\title{
Síncope como forma de debut de la estenosis mitral
}

\section{The syncope as a debut form of mitral stenosis}

Rodolfo Vega-Candelario, ${ }^{1, a}$

\section{Resumen}

Introducción: El mecanismo común a todo síncope es una alteración transitoria del flujo sanguíneo cerebral, que provoca una disminución momentánea del metabolismo cerebral, con afectación del sistema reticular ascendente y córtex cerebral. El síncope por estenosis mitral, no aparece en la literatura revisada.

Objetivos: Presentar un paciente con estenosis mitral que debuta con cuadro de síncope recurrente.

Caso clínico: Paciente de 45 años, mestizo, sin antecedentes de enfermedad, trabajador agrícola, ingresa en sala de medicina interna por padecer síncopes recurrentes. Fue valorado por neurólogo y cardiólogo; se diagnosticó estenosis mitral crítica con hipertensión pulmonar grave y disfunción sistodiastólica de ventrículo derecho. Luego de la sustitución valvular mitral por cirugía cardiovascular no volvió a padecer episodios sincopales y lleva una vida normal biopsicosocial y laboral.

Comentarios: La estenosis mitral crítica ocasiona disminución del llenado ventricular y disminución del gasto cardiaco con disminución del flujo sanguíneo cerebral, acentuado por la hipertensión pulmonar grave insuficiencia cardiaca derecha. Ocasionó síncopes repetitivos.

Palabras clave: síncope, estenosis mitral, hipertensión pulmonar, gasto cardiaco, flujo sanguíneo cerebral.

Abstract

Introduction: The common mechanism to the syncope is a transient alteration in cerebral blood flux, this fact generate a temporary decrease in the brain metabolism with involvement of the ascending reticular system and cerebral cortex. The syncope due to mitral stenosis does not appear in the reviewed literature.

Objectives: To show a clinical case from a patient with mitral stenosis that debuts with recurrent syncope.

Clinical case: A 45-year-old patient, mestizo without history of disease, a farmer, is admitted to the interna medicine ward for recurrent syncope. He was evaluated by a neurologist and a cardiologist; he was diagnosed with critical mitral stenosis with severe pulmonary hypertension and a right ventricular Systo-diastolic dysfunction. After the mitral valve replacement by cardiovascular surgery, he did not suffer syncopal episodes again and leads a normal biopsychosocial and work life.

Comments: The critical mitral stenosis causes decreased in the ventricular filling and decreased the cardiac output with decreased cerebral blood flux, accentuated by the severe pulmonary hypertension and the right heart failure. This fact caused repetitive syncope.

Keywords: syncope, mitral stenosis, pulmonary hypertension, cardiac output, cerebral blood flow.

${ }^{1}$ Profesor asistente de la Facultad de Ciencias Médicas de Ciego de Ávila, Cuba.

${ }^{a}$ Cardiólogo

ORCID

https://orcid.org/0000-0003-4459-8350

\section{Correspondencia a:}

Rodolfo Vega Candelario

Hospital Provincial Docente "Roberto Rodríguez Fernández, Morón, Ciego de Ávila, Cuba.

Email: rvc_50@infomed.sld.cu

Fecha de recepción: 02 de febrero de 2020

Fecha de aprobación: 18 de marzo de 2020

Citar como: Vega-Candelario R Síncope como forma de debut de la estenosis mitral. Rev. Peru. Investig. Salud. [Internet]; 4(2): 83-86. Available from:

http://revistas.unheval.edu.pe/index.php /repis/article/view/618

2616-6097/@2020. Revista Peruana de Investigación en Salud. Este es un articulo Open Access bajo la licencia Permite copiar y redistribuir el material en cualquier medio o formato. Usted debe dar crédito de manera adecuada, brindar un enlace a la licencia, e indicar si se (c) (7)

\section{Introducción}

El mecanismo común a todo síncope es la alteración transitoria del flujo sanguíneo cerebral, que provoca disminución momentánea del metabolismo cerebral, con afectación del sistema reticular ascendente y córtex cerebral, lo cual origina disminución del nivel de conciencia. Por experiencias en la mesa basculante, la caída de la presión arterial media por debajo de $60 \mathrm{mmHg}$, provoca una alteración del mecanismo de autorregulación del flujo cerebral, con la consiguiente aparición de síncope. Si esta pérdida de flujo cerebral se mantiene por encima de un tiempo crítico, en torno a 5 minutos, puede ocasionar lesiones neurológicas irreversibles. Las causas de síncope son numerosas, no obstante, es preciso hacer el diagnóstico diferencial con otras causas de disminución de conciencia, que no se consideran síncopes, como los accidentes isquémicos transitorios (AIT) vértebro basilares, trastornos metabólicos como la hipoglucemia, hipoxia, intoxicaciones, etc. Existen dos grandes tipos de síncope: los de origen cardiovascular y los neuromediados (14).
El tratamiento del síncope debe ir orientado a solventar la causa subyacente y evitar recidivas. Los síncopes cardiogénicos deberán ser tratados por los especialistas, con tratamientos específicos (marcapasos, desfibriladores, fármacos, cirugía, intervencionismo cardiaco). En el caso de los neuromediados, las medidas higiénicas y posturales deben ser el primer paso terapéutico. Otros tratamientos (fármacos, marcapasos) no han demostrado grandes beneficios (4-9).

Se conocen muchas causas de síncope, pero en ninguna aparece la estenosis mitral, menos como único síntoma. Este debut es frecuente en la estenosis aórtica, tumores cardiacos primarios y secundarios, bloqueos aurículoventriculares de segundo y tercer grados, enfermedad del nódulo sinusal, arritmias cardíacas (sobre todo ventriculares), taponamiento cardiaco, hipertensión pulmonar primaria, síndrome de Brugada, síndrome de QT prolongado, pero no en la estenosis mitral (9-13).

Los síncopes de origen cardiovascular (14-16) son frecuentes (3 al $5 \%$ de urgencias atendi- 
das; 1 al $2 \%$ de ingresos en un hospital). El 20 $\%$ de la población sufre un síncope al menos una vez en su vida. El pronóstico es muy variable y está de acuerdo con la causa. Los de origen cardiaco presentan una mortalidad estimada entre el $18 \%$ y $33 \%$ anual. El principal predictor de mortalidad enlos pacientes con síncope, es la presencia de cardiopatía estructural (10).

El paciente que se describe en este trabajo, ingresó con el cuadro de síncope, sin antecedentes patológicos familiares ni personales de cardiopatías, ni síntomas que la recuerden.

El objetivo del trabajo es presentar un paciente con estenosis mitral que debuta con cuadro de síncope recurrente.

\section{Caso clínico}

Paciente de 45 años de edad, mestizo, con buena salud anterior, sin hábitos tóxicos, obrero agrícola, que desde hacía 6 meses sufría síncopes, con pérdida total de la conciencia. Los familiares señalan que las convulsiones tónico clónicas generalizadas duran de 30 a 35 minutos incluyendo período post ictal del síncope convulsivo, luego el paciente se recupera, no recuerda nada y sigue trabajando, en la mayor parte de las ocasiones relacionado con esfuerzos físicos y raras veces sin relación con el mismo.

Fue valorado por el clínico, el neurólogo y el cardiólogo; se realizó tomografía axial computarizada (TAC) simple y contrastada de cráneo, electrocardiograma, electroencefalograma, telecardiograma y estudio hematológico completo. Los resultados fueron normales. Después de un minucioso interrogatorio $y$ examen físico, se diagnostica una estenosis mitral crítica, con hipertensión arterial sistólica pulmonar grave. Se realizó ecocardiograma Doppler a color y se encontró disfunción sistodiastólica de ventrículo derecho. Era lógico considerar lo anterior como causa del síncope.

El uso de inhibidores de la fosfodiesterasa 5 en la cirugía cardiaca, con hipertensión arterial pulmonar, está en estudio por la necesidad de prevenir complicaciones posquirúrgicas y aparición del fallo de ventrículo derecho (VD) (15-18); que en el enfermo ya estaba presente. Se comenzó con una dosis de $50 \mathrm{mg}$ diarios y se incrementó hasta $50 \mathrm{mg}$ cada 8 horas, según el resultado ecocardiográfico. Al cabo de 6 meses, se intervino quirúrgicamente con sustitución valvular mitral mecánica a corazón abierto en el Instituto de Cardiología y Cirugía Cardiovascular de Cuba. La evolución postoperatoria fue excelente. Después del posoperatorio se mantuvo con $150 \mathrm{mg}$ de sildenafilo durante 6 meses, asintomático, sin síncope, además de warfarina, con INR (por sus siglas en inglés) (International Normalized Ratio) (en español la Proporción Normalizada Internacional) entre 2 y 3.

\section{Comentarios}

Aunque la estenosis mitral no se relaciona como causa de síncope, este enfermo sufrió síncopes, con convulsiones tónico-clónicas generalizadas recurrentes.

En este enfermo, la estenosis mitral (Fig. 1,2,3) sí pudo ser causa de síncope, por el grado de estenosis y sus repercusiones sobre la presión arterial pulmonar, la insuficiencia tricúspidea y la insuficiencia cardíaca derecha (15-18).

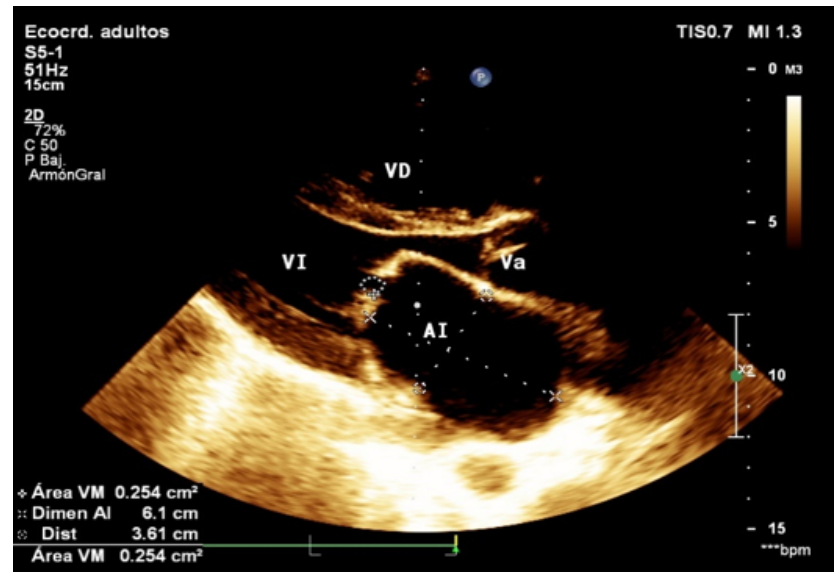

Leyenda: Al (aurícula izquierda); VI (ventrículo izquierdo); Va (válvula aórtica sana); VD (ventrículo derecho).

Fig. 1 - Ecocardiograma bidimensional en eje largo paraesternal izquierdo. Muestra una aurícula grande, con dificultad de apertura de la válvula mitral. EI área valvular mitral es de $0,25 \mathrm{~cm} 2$, igual que en eje corto.

La estenosis mitral crítica, fisiopatológicamente ocasiona disminución del llenado ventricular izquierdo. Esto se debe a una dificultad mecánica a nivel de dicha válvula al paso de la sangre que proviene de la aurícula izquierda y, por ende, disminución del gasto cardiaco. Además, la hipertensión pulmonar grave secundaria, ocasiona disminución del 
intercambio gaseoso, hipoxemia por aumento de la resistencia y fibrosis de la barrera hematoalveolar que ocasiona un círculo vicioso y como tal puede ser causa de síncope. Las consecuencias de la insuficiencia cardíaca derecha, la insuficiencia tricúspidea y la dificultad de perfusión hematoalveolar, constituyen un cuadro multifactorial que explica la disminución del flujo cerebral, con consecuencias dramáticas a nivel de la sustancia reticular, la corteza cerebral y la justificación del síncope cardiaco por estenosis mitral crítica (15-18) (Fig. 1,2). La hipertensión sistólica de arterial pulmonar (57 $\mathrm{mmHg}$ ) contribuye al síncope convulsivos recurrente (fig. 3).

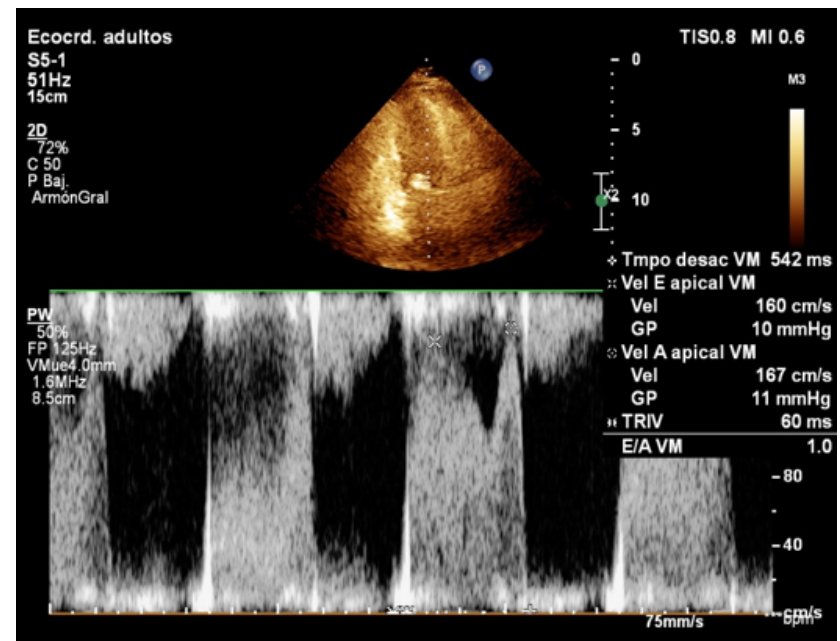

Fig. 2 - Ecocardiograma Doppler de válvula mitral (VM). Muestra los elementos auscultatorios de los cambios del ciclo cardiaco en la estenosis mitral crítica.

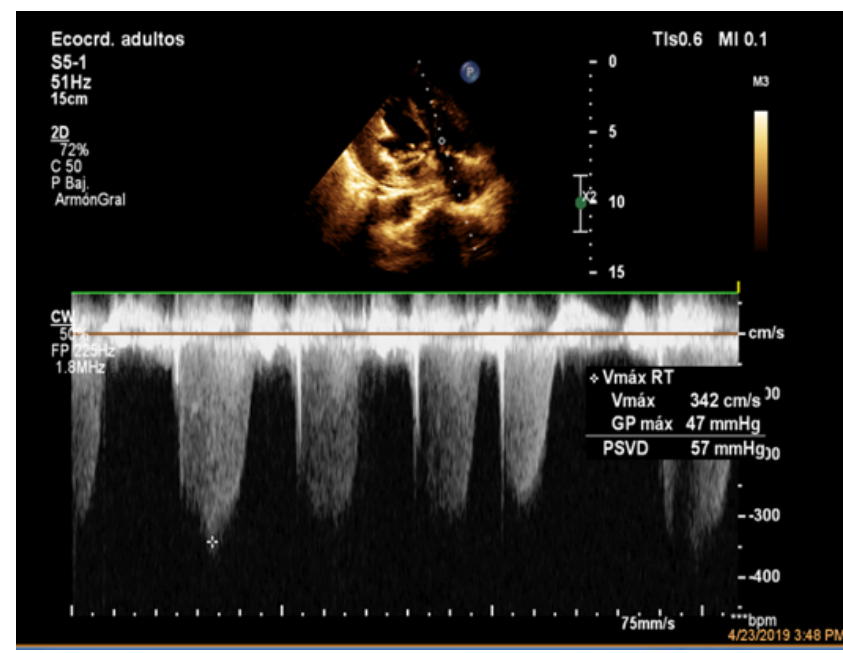

Fig. 3 - Eje 4 cámaras desde el ápex de ecocardiograma doppler. Se observa una elevación de las presiones en el ventrículo derecho que infiere la hipertensión pulmonar grave: Gradiente de presión máximo de 47 $\mathrm{mmHg}$ y Presión sistólica del Ventrículo Derecho de 57 mm Hg
El sildenafilo fue efectivo sobre la hipertensión arterial pulmonar grave y con eficacia pre quirúrgica y post quirúrgica de la cardiopatía adquirida (estenosis mitral crítica) (15-18).

\section{Referencias}

1. Lacunza Ruiz FJ, García Alberola JR, Gimeno Blanes M, Valdés M. Síncope. Unidades de Síncope y Arritmias. Medicine. 2005[acceso: 12/11/2019]; 9(37):2447-54. Disponible en:

http://www.sciencedirect.com/science/arti cle/pii/S0211344905737425/

2. Moya Mitjans A, Rivas Gandara N, Sarrias Merce A, Pérez Rodón J, Roca Luque I. Puesta al día: Arritmias (VIII), Síncope. Rev. Esp Cardiol. 2012 [acceso: 12/11/2019]; 65(8):755-65. Disponible en: https://www.revespcardiol.org/es-pdfS0300893212001947

3. Matthew JR, David E, Andrew J, Robin RJ, Prescott KG, Keith GJ, Gray R. The ROSE (risk stratification of syncope in the emergency department) study. Journal of the American College of Cardiology. 2010 [acceso: 15/11/2019]; 55(8) 713-21. Disponible en:

http://www.onlinejacc.org/content/accj/55/ 8/713.full.pdf

4. Tretter JT, Rae Ellen WK. Distinguiendo el síncope cardiaco del síncope. J Pediatr. 2013 [acceso: 2/12/2019]; 163: 1618-23. Disponible en:

https://www.jpeds.com/article/S00223476(13)00881-0/pdf

5. Azocar D, Ruiz Granell R, Ferrero A, Martínez Brotons $A$, Izquierdo $M$, Domínguez E, et al. Síncope y bloqueo de rama. Rendimiento del uso escalonado del estudio electrofisiológico y de la monitorización electrocardiográfica prolongada. Rev. Esp Cardiol. 2011 [acceso: 12/12/2019]; 64(3): 213-9. Disponible en:

http://www.revespcardiol.org/es/content/ar ticulo/90000664/

6. Moya A, Sutton R, Ammirati F, Blanc JJ, Brignole M, Johannes BD, et al. Guía de práctica clínica para el diagnóstico y manejo del síncope (versión 2009). Rev Esp Cardiol. 2009 [Acceso: 20/12/2019]; 62(12): 1466. Disponible en:

https://www.revespcardiol.org/es-pdfS0300893210000825

7. Shen WK, Decker WW, Smars PA, Goyal DG, Walker AE, Hodge DO, et al. Syncope 
Evaluation in the Emergency Department Study (SEEDS): a multidisciplinary approach to syncope management. Circulation. 2004 [acceso: 25/12/2019]; 110: 3636-45. Disponible en:

https://www.ahajournals.org/doi/pdf/10.11 61/01.CIR.0000149236.92822.07

8. Farwell DJ, Sulke AN. Does the use of a syncope diagnostic protocol improve the investigation and management of syncope? Heart. 2004 [acceso: 27/12/2019]; 90(1):52-8. Disponible en:

https://www.ncbi.nlm.nih.gov/pmc/articles/ PMC1768011

9. Ammirati F, Colivicchi F, Santini M. Diagnosing syncope in clinical practice: implementation of a simplified diagnostic algorithm in a multicenter prospective trial. Our Heart J. 2000 [access: 28/12/2019]; 21(11): 935-40. Disponible en:

https://www.revespcardiol.org/es-pdf13145649

10. Brignoles $M$, Ungar A, Bartoletti A, Ponassi I, Lagi A, Mussi C, et al. Standardized-care pathway vs. usual management of syncope patients presenting as emergencies at general hospitals. Euro pace. 2006 [acceso: 30/12/2019]; 8(8): 644-50. Disponible en:

http://asmn.netribe.it/asmnbiblio2015/pub blicazioni/Europace\%202006.pdf

11. Rodríguez Entem F, González Enríquez S, Juan J, Olalla $A$, Cobo Beláustegui $M$, Expósito García V, et al. Manejo del síncope en el servicio de urgencias sin ingreso hospitalario. Rev. Esp Cardiol. 2008 [acceso: 04/01/2020]; 61(1): 22-8. Disponible en:

https://www.revespcardiol.org/es-pdf13114953

12. Moya A, Rivas N, Pérez Rodón J, Roca I, García Dorado D. El síncope: un problema con mayúsculas. Rev. Esp Cardiol Supl. 2010 [acceso: 6/01/2020]; 10(Supl.1):53A$59^{a}$. Disponible en:

https://www.sciencedirect.com/science/ article/abs/pii/S11313587 10701126
13. Prieto Salcedo ML, Pérez López AM. Protocolos de Cardiología Síncopes. Bol. Pediatr. 2006 [acceso: 13/01/2020]; 46 (Supl 2): 281-5.Disponible en: http://www.sccalp.org/boletin/46_supl2/Bol Pediatr2006_46_supl2_281-285.pdf

14. Ramakrishna G, Sprung J, Ravi B, Chandrasekaran K, McGoon M. Impact of pulmonary hypertension on the outcomes of noncardiac surgery: Predictors of perioperative morbidity and mortality. J Am Coll Cardiol. 2005[acceso: 15/11/2020]; 45(10):1691-9. : Disponible en:

https://www.sciencedirect.com/science/art icle/pii/S0735109705004961?via\%3Dihub

15. Galie N, Olschewski H, Oudiz R, Torres F, Frost A. Ambrisentan for the treatment of pulmonary arterial hypertension. Circulation. 2008 [acceso: 12/01/2019]; 117(23): 3010-9. Available from:

https://www.ncbi.nlm.nih.gov/pubmed/185 06008

16. Lewis G, Lachmann J, Camuso J, Lepore $J$, Shin J, Martinovic M, et al. Sildenafil improves exercise hemodynamics and oxygen uptake in patients with systolic heart failure. Circulation. 2007 [acceso: 15/01/2020]; 115(1): 59-66. Disponible en: https://pdfs.semanticscholar.org/2f04/979 0063265fb62706efd30573fada2ac0af3.pd $f$

17. Hemnes A, Champion H. Right heart function and hemodynamics in pulmonary hypertension. Int J Clin Pract 2008 [acceso: 18/01/2020]; 62(s160): 11-9. Disponible en: https://onlinelibrary.wiley.com/doi/pdf/10.1 111/j.1742-1241.2008.01812.x

18. Falk V, Bax JJ, De Bonis M, Hamm C, Holm H, Lung B. Guía ESC/EACTS 2017 sobre el tratamiento de las valvulopatías. Rev Esp Cardiol. 2018; 71(2): 110.e1-e47. Disponible en:

https://www.revespcardiol.org/es-pdfS0300893217308096 